\title{
Thoughts on being a senior radiologist
}

\author{
Brian D. Coley ${ }^{1}$
}

Published online: 5 January 2021

(C) The Author(s), under exclusive licence to Springer-Verlag GmbH, DE part of Springer Nature 2021

An article by Drs. Markowitz, Taylor and Darge [1] in this issue of Pediatric Radiology makes a solid case for the value that senior radiologists can make to an academic department. This is a complex issue that raises a number of questions that are worthy of additional exploration, realizing that some answers are not absolute and that different departments have different needs, and that solutions are not always universally applicable.

In the article, the authors make a financial argument about a per diem system of coverage provided by senior radiologists officially retired from their university system. By not taking the benefits provided to regular employed faculty (and depending upon the negotiated wage), per diem radiologists can indeed cost a department less per shift. The flexibility that per diem staff can have in scheduling for vacations, meetings and unexpected faculty absences significantly eases the unenviable job of the department scheduler and the rest of the faculty. The reported productivity numbers make a convincing argument that per diem senior radiologists can earn their keep. The argument that such an arrangement can lead to increased academic output is less convincing, but with just 6 months of data this cannot be expected to be proved. Given that radiologists' salaries are well above National Institutes of Health salary caps, a financial argument that per diem radiologists significantly foster research is unlikely to be persuasive, although the general point that per diem workers overall cost less than the faculty members who are given time to pursue research still holds.

The cultural benefit that senior radiologists bring to a department is difficult to quantitate, but I agree with the authors that there is value. Knowledge, perspective and occasionally wisdom (not a guarantee with age, despite popular adages) of senior radiologists can contribute to the intellectual life of a

Brian D. Coley

brian.coley@cchmc.org

1 Department of Radiology, Cincinnati Children's Hospital Medical Center, University of Cincinnati College of Medicine,

3333 Burnet Ave., Cincinnati, OH 45229, USA department and the education of younger trainees and faculty. Senior radiologists' presence sends a positive message to the entire faculty that the department values its long-term professors and their expertise, and is open to helping them to contribute in whatever ways they can.

Potential downsides of the authors' per diem arrangement certainly exist and are acknowledged. Being allowed to opt out of call or less desirable weekend and evening hours might be viewed as an earned right, but depending upon group size and culture this could lead to increased burdens on regular faculty and have the potential to foment resentment. Understandably, the per diem faculty read fewer CT and MRI examinations (while nonetheless keeping pace in terms of total work relative value units generated). Keeping the radiography list under control can certainly reduce pressure on those doing the complex cross-sectional imaging. However, the pressure of always looking at complicated cases without the respite provided by simpler and often more straightforward cases can exacerbate cognitive load and mental toil on the regular faculty. While flexibility in scheduling and lower cost can be an advantage for the per diem model, at some point hiring faculty members who can fill a wider variety of shifts and work effectively across more modalities might better serve a department's needs and become preferable.

The authors touched upon the thorny issue of age and cognitive decline. While not the primary focus of their paper, it is nonetheless a part of the bigger picture. Unlike some professions, most physicians are not subject to mandatory retirement ages and only a few academic institutions have mandatory age-related cognitive testing. By some measures, human cognitive performance begins to decline in the mid-20s (meaning that radiologists are on the decline before we even finish residency) and visual and auditory declines are measurable in the $40 \mathrm{~s}$ and 50s. Fortunately, other aspects of age such as experience, accumulated clinical knowledge, lesser tendency to overcall findings, and the ability to make meaningful integrated interpretations of imaging studies can increase. Cognitive ability does not necessarily equate with clinical competency (something very difficult to measure), and competency can 
have different definitions depending upon the task being evaluated. While age-related competency is not to be ignored and is likely to be an ongoing point of discussion, arbitrary determinations of competency dependent solely upon physician years is probably not defensible.

The physician workforce is aging. Late-career radiologists are defined as those older than the age of 56 years (I suspected that I was in fact a late-career radiologist but did not necessarily appreciate having this confirmed...). Physician shortages in the coming years seem guaranteed, and pediatric radiology is already facing them. Given this, every capable pediatric radiologist can be viewed as an important health care resource. To continue to provide the imaging care that our patients deserve, our specialty needs to make the most of our available workforce while at the same time recognizing the need to mitigate the pressures of increasing volumes and cognitive loads that are part of job dissatisfaction and burnout. Making the most of our available pediatric radiology talent is important now and will only become more necessary. Providing part-time work with benefits for faculty with family care demands, accommodating those wanting to be less than a fulltime equivalent because of physical constraints or as a defense against burnout, providing for those looking for a gradual decrement in clinical responsibilities as they head toward retirement, or making space for the senior radiologist who still has the skill and desire to contribute are all ways that departments can meet their clinical demands and maintain the excellence of imaging care that we all expect of ourselves.

I hope my eventual successor will be willing to meet with me to discuss....

\section{Compliance with ethical standards}

Conflicts of interest None

\section{Reference}

1. Markowitz RI, Taylor GA, Darge K (2020) The impact of per diem senior pediatric radiologists in an academic setting. Pediatr Radiol. https://doi.org/10.1007/s00247-020-04886-9

Publisher's note Springer Nature remains neutral with regard to jurisdictional claims in published maps and institutional affiliations. 